\title{
NLPDove at SemEval-2020 Task 12: Improving Offensive Language Detection with Cross-lingual Transfer
}

\author{
Hwijeen Ahn ${ }^{1 *}$, Jimin Sun ${ }^{2 *}$, Chan Young Park ${ }^{3^{*}}$, Jungyun Seo ${ }^{1}$ \\ ${ }^{1}$ Sogang University, Republic of Korea \\ ${ }^{2}$ Seoul National University, Republic of Korea \\ ${ }^{3}$ Language Technologies Institute, Carnegie Mellon University, USA \\ \{hwijeen, seojy\}esogang.ac.kr, \\ jiminsunedm.snu.ac.kr, chanyoundes.cmu.edu
}

\begin{abstract}
This paper describes our approach to the task of identifying offensive languages in a multilingual setting. We investigate two data augmentation strategies: using additional semi-supervised labels with different thresholds and cross-lingual transfer with data selection. Leveraging the semisupervised dataset resulted in performance improvements compared to the baseline trained solely with the manually-annotated dataset. We propose a new metric, Translation Embedding Distance, to measure the transferability of instances for cross-lingual data selection. We also introduce various preprocessing steps tailored for social media text along with methods to fine-tune the pre-trained multilingual BERT (mBERT) for offensive language identification. Our multilingual systems achieved competitive results in Greek, Danish, and Turkish at OffensEval 2020.
\end{abstract}

\section{Introduction}

Online social media has become one of the most important means of communication. Unfortunately, the discourse is often laden with abusive language that can have damaging effects on social media users (Zampieri et al., 2019a). As online abuse grows as a serious social problem, many social media platforms are employing human moderators to track inappropriate contents and comments. However, such human moderation comes at the cost of additional operation and the mental suffering of the workers (Roberts, 2014; Dosono and Semaan, 2019). In response, the automatic identification of offensive language has been a rising area of interest to both academia and industry. Computational methods presented in several prior studies (Park and Fung, 2017; Pitsilis et al., 2018; Wulczyn et al., 2017; Founta et al., 2018) have shown promising results on how we can automatically detect a large number of offensive contents with minimal human intervention.

As an effort to promote this line of research, OffensEval was organized at SemEval-2019 (Zampieri et al., 2019b). The organizers released a dataset, Offensive Language Identification dataset (OLID), that contains English tweets with human annotations over three sub-tasks: 1) whether a tweet is offensive or not, 2) whether an offensive tweet is targeted or not, and if so 3) whether the target is an individual or a group. The second edition of OffensEval, OffensEval 2020 : Multilingual Offensive Language Identification in Social Media, holds the same task but extends the OLID to multiple languages (English, Arabic, Danish, Greek, and Turkish) (Zampieri et al., 2020).

This paper describes our system, NLPDove, submitted to OffensEval 2020 . We present two different data augmentation strategies. First, for English, we augment the training set with reliable samples filtered out from the provided semi-supervised dataset. Second, we use samples from languages other than the target language to enhance performance in the target language, namely cross-lingual transfer. Specifically, we propose a new metric, Translation Embedding Distance(TED), to measure the data transferability of each example in the data. We use the metric to effectively select additional training data samples from other available transfer languages. We also introduce various preprocessing steps to extract meaningful information from user-generated texts despite their inherently noisy nature. Our model is based on the

\footnotetext{
This work is licensed under a Creative Commons Attribution 4.0 International License. License details: http:// creativecommons.org/licenses/by/4.0/.

* The first three authors contributed equally.
} 


\begin{tabular}{lcccccc}
\hline Language & Train size & Valid size & OFF:NOT ratio & & \\
\cline { 1 - 4 } \cline { 5 - 6 } Arabic & 7202 & 800 & 0.25 & & Preprocessing & \# of Cases \\
Danish & 2666 & 296 & 0.15 & & Emoji substitution & 1454 \\
English & 12691 & 1411 & 0.49 & & Hashtag segmentation & 2290 \\
Greek & 7871 & 874 & 0.40 & & Letter casing normalization & 12665 \\
Turkish & 28582 & 3176 & 0.24 & & URL replacement & 2140 \\
English-semi & $9 \mathrm{M}$ & - & - & & Punctuation trimming & 504 \\
\hline
\end{tabular}

Table 1: Summary statistics of the dataset. Englishsemi denotes the semi-supervised English dataset. For English, we also used the OLID from OffensEval 2019 (Zampieri et al., 2019a). We randomly split the Table 2: Preprocessing methods and the number of examples modified by each method in the English training data. provided data into train and validation set by 9:1.

multilingual pre-trained language model, particularly multilingual BERT (mBERT) (Devlin et al., 2019). Our final submitted model is an ensemble of multiple mBERT models with different pooling mechanisms, representation layers and randoms seeds. Our submission achieved macro F1 of 0.85 in Greek (1st/38), 0.79 in Danish (3rd/40), 0.80 in Turkish (5th/47), 0.91 in English (15th/86), and 0.80 in Arabic (20th/54). 1

In the remainder of this paper, we first describe the dataset and the task of OffensEval $2020(\S 2)$. We then present our method, including the data augmentation strategies, preprocessing pipeline, and model architecture $(\S 3)$. The evaluation results of our final submission are reported along with other experiments to further investigate the effectiveness of our method ( $(4)$. We then discuss our findings and negative results to shed light on the limitations and remaining challenges in the current offensive language identification models $(\S 5)$. Finally, we review related work $(\S 6)$ and conclude (§7).

\section{Data}

The most noticeable update in OffensEval 2020 is the extension of the task to four additional languages other than English: Arabic (Mubarak et al., 2020), Danish (Sigurbergsson and Derczynski, 2020), Greek (Pitenis et al., 2020), and Turkish (Çöltekin, 2020). This change facilitates the research of offensive language identification in multiple languages, and also enables researchers to explore the feasibility of cross-lingual approaches of the task. One thing to note is that unlike English tweets which had annotations for all three sub-tasks, the four new languages do not have all of them; they are only annotated on the first sub-task, which tells whether they are offensive or not.

In the case of English, in addition to the manually annotated tweets from the previous workshop (Zampieri et al., 2019a), OffensEval 2020 released a large semi-supervised dataset where samples are labeled by a set of trained models, not by human annotators (Rosenthal et al., 2020). Therefore, the labels are not binary but given as floating-point numbers between 0 and 1 (i.e. the average score of multiple models), with their standard deviation indicating the confidence level among classifiers. For further details regarding the data, we refer to the original paper (Rosenthal et al., 2020). The summary statistics of the dataset are presented in Table 1.

\section{Method}

There are three important components in our system: training data augmentation, extensive preprocessing, and model ensembling. The following subsections describe each part in detail.

\subsection{Data Augmentation}

mBERT and other large-scale pre-trained language models are known for their massive number of parameters (Conneau and Lample, 2019). Thus, they require a substantial amount of data to be fine-tuned

\footnotetext{
${ }^{1}$ The code for our system can be found at https://github.com/hwijeen/OffensEval2020
} 
sufficiently to the downstream task. And yet, the dataset in hand may not be large enough, especially for some languages due to the expensive annotation process. To alleviate this data sparsity issue, we propose two data augmentation approaches to make most of the provided semi-supervised labels and multilingual data.

Semi-supervised Labels It has become a common practice to augment training data with machine annotated or synthetic labels when the training data is insufficient (Sennrich et al., 2016). There is almost an infinite number of unlabeled Twitter data available, many of which contain offensive languages. One way to obtain a substantial amount of pseudo-labels is to predict unlabeled tweets with models trained with existing labeled data. OffensEval 2020 released over nine million English tweets labeled in this semi-supervised manner (Rosenthal et al., 2020). Each sample was given with its average label probability from several trained models and the standard deviation of the probabilities which indicates the confidence level. One practical question lies in how to utilize this data while reducing the effect of noisy labels and controlling the data quality. One straightforward approach is to set certain thresholds to filter out the uncertain examples. We investigate different thresholding strategies with respect to the two measures, the average probability and the standard deviation.

Cross-lingual Data Selection Multilingual pre-trained language models allow seamless cross-lingual transfer; they map texts in different languages into the same representation space without introducing extra costs. Cross-lingual transfer can be especially helpful when the training data is limited for the target language, i.e., low-resource settings. Transferring examples from high-resource languages to low-resource languages often results in great improvements (Chen et al., 2018; Xu and Yang, 2017).

Although it is clear that cross-lingual transfer can be of help, the question of which data to additionally use remains an open problem. Should we use the entire dataset, or is there a subset better than the entire dataset? We hypothesize that not all samples in the same transfer language will be equally helpful to the target language task, leading to the conclusion that a carefully selected subset will give higher performance gain than using the whole dataset.

To address the how part of data selection, we propose a new metric, translation embedding distance (TED), to quantify the transferability of a data sample or a language. We first define a sample's transferability as how useful the transfer language sample is when transferred to the target language task. Here, we hypothesize that transferability as a concept correlates with translatability; if a sentence is easily translated to the target language, it is more likely to be a useful training sample. To measure TED, we follow the three steps: first, we (machine) translate the training samples in transfer languages into the target language. ${ }^{2}$ Then, we extract sentence embeddings for both the original sentence and its translation using mBERT. More specifically, we applied mean pooling over the second-to-last (penultimate) layer of mBERT and used it as the sentence embedding. Finally, we measure the L2 distance between the two representations and refer to it as the TED of the instance. The transferability of a language, which we define as language-level TED, is simply the average of instance-level TED of samples in the transfer language.

We can then rank transfer samples according to their TED scores and choose top- $k$ samples with the lowest distance. Likewise, to find the most transferable languages, we can select the top- $k$ languages based on their language-level TED scores. To verify our hypothesis that adding samples in other languages can enhance performance in the target language task, we investigate the effectiveness of both language-level and instance-level data selection in $\S 4.2$.

\subsection{Preprocessing}

User-generated contents are inherently noisy, whereas the pre-trained language models that we use throughout our system are mostly trained with clean texts such as Wikipedia articles and BookCorpus (Devlin et al., 2019). To minimize the discrepancy, we applied various preprocessing steps to normalize the noisy tweets provided in our dataset. In particular, we focus on normalizing the unique conventions of tweets (e.g. hashtags, mentions, emojis) and their irregular usage of punctuation and capitalization.

\footnotetext{
${ }^{2}$ We used Google Translate in our experiments.
} 
Table 2 lists all of the considered methodologies and the number of cases found for each method in the English data. Each item is explained in more detail below.

Emoji Substitution Although emojis are effective means of expressing sentiments, learning a good representation of emojis with neural models can be challenging due to their low frequency. Another challenge is that the most pre-trained language models are not acquainted with the use of emojis as the texts used to train them did not contain emojis. To fully benefit from the knowledge embedded in pre-trained language models, we replaced all emojis with their plain text descriptions (e.g. $\odot \rightarrow$ smiley face) using an off-the-shelf library ${ }^{3}$.

Hashtag Segmentation Hashtags are prevalent in tweets, and they often contain essential information and keywords. Conventionally, hashtags are prefixed with the \# symbol followed by phrases concatenated without whitespaces. In order to extract all relevant information from hashtags, we split them into words using a word segmentation toolkit ${ }^{4}$. We preserved the \# symbol in the beginning of segmented words to help the model differentiate hashtags from plain texts.

Letter Casing Normalization In social media texts, people often use capitalization to express the intensity of their emotions. For example, "GET OUT!" sounds much more intense than "get out!". Although "GET" and "get", in this case, possess the same meaning and only differ in the degree of emphasis, they will be assigned to completely different tokens if we do not use a canonical form representing both variants. Keeping the original form may be useful to preserve the richness in expressing emotional intensity, but the embedding quality of the less frequent variants cannot be assured. In this case, "GET" would occur much infrequently than "get" in the training corpus. Therefore, we first normalized these casing variations by lowercasing all words. But at the same time, we prepended a special token $\langle$ has_cap $\rangle$ when the word contains an uppercase alphabet and $\left\langle a l l_{-} c a p\right\rangle$ when the entire word was uppercased.

URL Replacement Tweets often include raw urls that redirect users to a reference page. In the provided data, most of the urls were already replaced with two special tokens, HTTP and URL. We unified them into HTTP, since HTTP is the token used during the pre-training stage of mBERT.

Punctuation Trimming One characteristic of social media text is the irregular usage of punctuation marks. For instance, the two sentences "I am sad.." and "I am sad........" differ slightly in their use of punctuation marks. The difference between the two sentences is negligible for a human reader in terms of sentiment. However, their sentence representations may differ significantly since the latter's representation would be diluted with its repeated use of periods. Hence, we limit the maximum number of consecutive punctuation marks to three occurrences.

\subsection{Model}

In our final submission, we used an ensemble of several different mBERT models. Here, we explain how we selected and ensembled the models.

mBERT mBERT is a multilingual extension of BERT which shares the same architecture with BERT (Devlin et al., 2019). The main difference is that mBERT was trained on the monolingual corpora in multiple languages with an extended vocabulary, which enables seamless cross-lingual transfer. The model can take inputs in various languages, and we can either jointly train a model with both the transfer and target language or perform zero-shot transfer where the model is trained only with the transfer language. mBERT is shown to be highly effective in both settings (Pires et al., 2019), implying the model's ability to learn a universal representation space that is shared across languages.

We took advantage of the multilinguality of mBERT and used it as the main model for our system for all languages. We added a pooling layer and a linear classifier on top of mBERT for the classification task. The pooling layer takes mBERT representations of each word and combines them into a fixed-size vector.

\footnotetext{
${ }^{3}$ https://github.com/carpedm20/emoji

${ }^{4}$ http: //www.grant jenks.com/docs/wordsegment/
} 


\begin{tabular}{c|c|c|c|c|c}
\hline \multirow{2}{*}{ Model } & \multicolumn{4}{|c}{ Language } \\
& Arabic & Danish & English & Greek & Turkish \\
\hline mBERT (Single model) & 0.8722 & $\mathbf{0 . 7 8 3 7}$ & 0.7770 & $\mathbf{0 . 8 3 4 9}$ & 0.7835 \\
Ensemble & $\mathbf{0 . 8 7 7 5}$ & 0.7514 & $\mathbf{0 . 7 8 2 2}$ & 0.8343 & $\mathbf{0 . 7 9 7 0}$ \\
\hline
\end{tabular}

Table 3: Performance of our system on development data. The ensemble model was used for final submission for all languages.

${ }^{5}$ We used the same hyperparameters values provided in Wolf et al. (2019) except for the learning rate, random seed, and dropout probability. For the three hyperparameters we tuned, we ran a grid-search to select the best configuration based on the validation set performance. We note that tuning the random seed, as found in Dodge et al. (2020), resulted in a significant performance gain.

Ensemble Model ensemble methods are widely employed to improve a model's robustness and generalization performance. With this motivation in mind, we developed a pipeline for model ensembling. We trained multiple BERT-based models for each task that differ by which BERT layer is used for the word representation (last, second-to-last) ${ }^{6}$, pooling mechanism (mean, max, concatenation of both, and $\mathrm{CNN}$ ), learning rate (1e-5, 1e-6), and also random seeds. We then chose the top three models according to the validation performance. In the case of English, we additionally considered an mBERT model trained with the augmented dataset that incorporates the semi-supervised dataset as described in $\S 4.2$. We used majority voting to aggregate the prediction of the selected models for the final prediction.

\section{Experiment Results}

\subsection{Main Results}

We used a macro-averaged F1 score as our main evaluation metric as in OffensEval 2020. Table 3 summarizes the performance of our models in the validation data for each target language. We also report the best performance of a single mBERT model for comparison. As explained in $\S 3.3$, our final submission models used a majority vote ensemble of several mBERT models.

\subsection{Data Augmentation Experiment}

Semi-supervised Labels For the English task, we used the human-annotated dataset from OffensEval 2019 as the primary data source and augmented it with the subset of the semi-supervised dataset from OffensEval 2020. As explained in Section 2, the semi-supervised labels are the averaged prediction scores of various trained models accompanied by the standard deviation of these scores.

One way to control the quality of the additional data is to filter out noisy data using the prediction confidence score of classifiers and their standard deviation. For instance, setting the minimum value of the offensive class confidence as 0.9 rather than 0.6 would result in a smaller but more reliable subset. Similarly, a sample with a lower standard deviation indicates a higher likelihood of multiple models agreeing to its predicted label, thereby more reliable. We performed a grid search over combinations of threshold values for offensive and non-offensive tweets as well as the standard deviation. In particular, we set the grid with three different threshold parameters: $t_{\text {off }} \in\{0.8,0.9\}, t_{n o t} \in\{0.2,0.3\}, t_{\text {std }} \in$ $\{0.1,0.125\}$. Data samples are selected when the standard deviation is lower than $t_{s t d}$, and its score is either higher than $t_{\text {off }}$ or lower than $t_{\text {not }}$.

The results are summarized in Table 4. Although varying the threshold resulted in additional data of different qualities and sizes, the difference in results was marginal. When about $30 \mathrm{~K}$ samples were added with cut-off values of $t_{o f f}=0.8, t_{n o t}=0.2, t_{s t d}=0.125$, we observed a small performance gain compared to others. In our final ensemble model, we included the models that performed better than the baseline which was trained solely on the human-annotated dataset.

\footnotetext{
${ }^{5}$ We experimented with mean pooling, max pooling, concatenation of both, and CNN pooling.

${ }^{6}$ It has been reported that BERT outputs from different layers encode different kinds of information (Kovaleva et al., 2019).
} 


\begin{tabular}{c|c|c|c|c}
\hline \multicolumn{3}{c|}{ Threshold } & $\begin{array}{c}\text { Additional } \\
\text { data size }\end{array}$ & Macro \\
OFF & NOT & std & (1) \\
\hline 0.8 & 0.2 & 0.1 & 3533 & 0.7693 \\
0.8 & 0.2 & 0.125 & 29681 & $\mathbf{0 . 7 7 9 3}$ \\
0.8 & 0.3 & 0.1 & 7956 & 0.7716 \\
0.8 & 0.3 & 0.125 & 83782 & 0.7738 \\
0.9 & 0.2 & 0.1 & 2769 & 0.7741 \\
0.9 & 0.2 & 0.125 & 19590 & 0.7709 \\
0.9 & 0.3 & 0.1 & 7192 & 0.7707 \\
0.9 & 0.3 & 0.125 & 73691 & 0.7453 \\
\hline \multicolumn{3}{c|}{ Baseline } & 0 & 0.7754 \\
\hline
\end{tabular}

Table 4: Results of data augmentation from semi-supervised labels. F1 scores are measured over the validation split of English data.

\begin{tabular}{c|c|c|c|c|c}
\hline Train data & $d a$ & $d a+e n_{\text {all }}$ & $d a+e n_{\text {top }}$ & $d a+e n_{\text {bottom }}$ & $d a+e n_{\text {rand }}$ \\
\hline Macro F1 & 0.77 & 0.78 & $\mathbf{0 . 8 4}$ & 0.72 & 0.72
\end{tabular}

Table 5: Results of cross-lingual data augmentation on the Danish validation data. $d a$ refers to the original Danish training data, and $e n_{\text {all }}$ is the entire English training dataset. $e n_{t o p}, e n_{\text {bottom }}, e n_{\text {rand }}$ are the subsets of samples according to the TED.

Cross-lingual Data Transfer We first consider language-level cross-lingual transfer strategies. That is, we seek the most effective transfer language for the given target language. To this end, we measure language-level TED for each transfer and target language pair. We then pick top- $k$ languages that have high language-level TED score for each target language.

Cross-lingual transfer has been shown to be effective especially when the target language dataset is not sufficient. Our experiments focus on the setting where Danish is the target language as it has the least number of training samples. $k$ was set to 1 for language selection. Results showed that English (9.83) is the most useful transfer language to Danish, followed by Arabic (10.85), Greek (22.02) and Turkish (23.08). The high transferability from English to Danish is probably due to the two languages' surface-level resemblance, which comes from the shared orthography. We note that English turned out to be the most effective language to Danish even in terms of zero-shot transfer performance (trained only on the source language and tested on the target language).

Next, we consider the transfer strategy on a more fine-grained instance-level. We construct a subset of the dataset of the most transferable language based on each sample's transferability. Specifically, we sort the English samples based on their TED to Danish, and choose the samples with the lowest TED. We investigate three different ways to build the subset: taking top/bottom/random- $k$ English samples ( $e n_{t o p}$, $e n_{\text {bottom }}, e n_{\text {rand }}$ ). In our experiment, we set $k$ to 1300 , which is about half the size of the Danish dataset. We compare the results with two baselines, one trained only with Danish data $(d a)$ and another one trained with the aggregated dataset of Danish and all English samples $\left(d a+e n_{\text {all }}\right)$. The results are in Table 5.

Results confirmed our hypothesis that we can further improve cross-lingual transfer by selecting samples that are more transferable than others. $d a+e n_{t o p}$ showed the best performance, while $d a+e n_{\text {bottom }}$ and $d a+e n_{\text {rand }}$ performed worse than $d a+e n_{\text {all }}$. This shows that naïvely using all samples in the transfer language (English) is not necessarily the best way to conduct cross-lingual transfer.

\section{Analysis and Discussion}

In this section, we analyze how cross-lingual data selection could help improve performance. In addition, we discuss limitations of the proposed metric, TED, and some negative results with masking offensive 


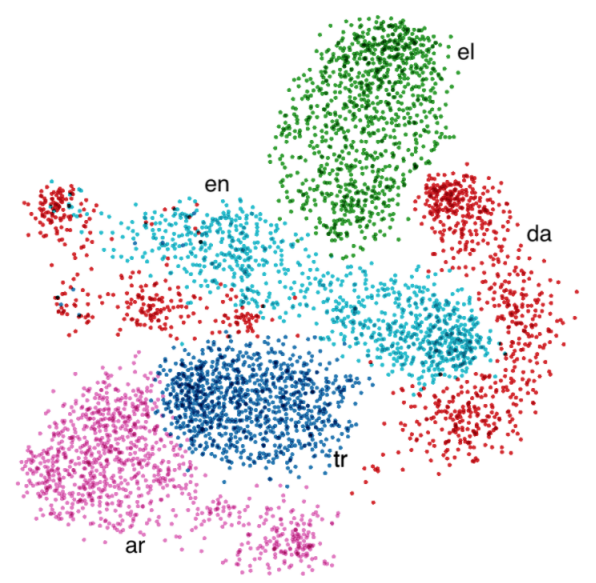

Figure 1: tSNE visualization of mBERT sentence embeddings of OLID samples. Samples are color coded according to their language (ar:Arabic, da:Danish, en:English, el:Greek, and tr:Turkish).

words with a hope to provide insights for future work.

\subsection{How Does Cross-lingual Data Selection Help?}

We showed that our data augmentation strategy using data from other languages helps improve the target task performance in Section 4.2. Here, we investigate why cross-lingual data selection was helpful.

We first take a look at the embedding space of mBERT to understand why language-level data selection, according to language-level TED, was beneficial. We randomly sampled a thousand samples from each language and visualized their sentence embeddings using tSNE (Maaten and Hinton, 2008) in Figure 1. From the visualization, we observed that the examples formed rough clusters based on their language. Interestingly, English and Danish clusters are placed near one another. This aligns with our experimental result in the previous section, where we found English to be the most transferable language to Danish by language-level TED. We speculate that this proximity in the embedding space may be related to the improvement yielded when English samples with low TED were added to the Danish dataset. mBERT might have benefited from the additional English data even while training a Danish classifier because the examples were relatively close to each other in the shared embedding space.

In addition, we manually inspect the examples with low TED scores which we assumed to be more transferable. Examples in Table 6 reveal that the samples with low TED are the ones that did not change significantly after the translation procedure. These samples often contain words used in both languages colloquially (e.g., creepy, fool), and tend to be short with a rather simple grammatical structure. We posit that this simplistic nature of the samples with low TED made themselves more translatable, thus more transferable, and led to improvements via cross-lingual transfer.

\subsection{Limitations of TED}

TED measures the transferability of an example with the help of a machine translation system. Incorporating an external off-the-shelf translator is convenient in that we do not need to build an additional translation system for multiple pairs of languages. However, still, the computation of TED comes with a cost of translating all samples for all possible transfer-target language pairs. Another limitation of our method is that the quality of data selection relies on the performance of the employed machine translation system. This dependency may lead to the misrepresentation of transferability in low-resource language samples because the quality of translation systems in these languages are generally deficient. We believe that the different approaches commonly used for cross-lingual transfer (e.g., alignment methods) may pave the way for building a transferability metric that does not rely on external translation modules. 


\begin{tabular}{|c|c|}
\hline Original (English) & Translated (Danish) \\
\hline @USER Creepy & @USER Creepy \\
\hline @USER Fool! & @USER Fool! \\
\hline $\begin{array}{l}\text { \#RestoreHumanity \#AntiFa September 22: Stop } \\
\text { the fascist NVU in \#Amsterdam: URL HT } \\
\text { @USER }\end{array}$ & $\begin{array}{l}\text { \#RestoreHumanity \#AntiFa September 22: Stop } \\
\text { det fascistiske NVU in \#Amsterdam: URL HT } \\
\text { @USER }\end{array}$ \\
\hline @USER I'M SO FU*KING READY & @USER I'M SO FU*KING KLAR \\
\hline $\begin{array}{l}\text { @USER @USER so sad \#taketworeferenceswith- } \\
\text { moniqueandchloe x }\end{array}$ & $\begin{array}{l}\text { @USER @USER så trist \#taketworeferenceswith- } \\
\text { moniqueandchloe } \mathrm{x}\end{array}$ \\
\hline
\end{tabular}

Table 6: English samples with low TED when transferred to Danish. Most examples were not changed significantly after the translation.

\subsection{Offensive Words in Inoffensive Contexts}

In our pilot experiment, we fine-tuned BERT with the OLID from OffensEval 2019 (Zampieri et al., $2019 b$ ). The model's initial performance was around 0.80 macro F1, which is comparable considering that the best performance from OffensEval 2019 was 0.82 (Liu et al., 2019). Qualitative analysis on the false-positive cases, however, showed that the model tends to classify tweets that include offensive words as offensive, regardless of the context. For example, "@USER And the fact he called you a $b^{*}$ tch after you respectfully told him your preference.." was classified as offensive although the term "b*tch" was not used to express any aggression toward the listener. Offensive words are also often used for alternative motives: as a topic of the statement or even to emphasize a positive sentiment. We have found many cases in the dataset where offensive terms have not been used in a despicable manner (e.g., " $f *$ cking awesome"). However, since most offensive words are paired with aggressive intentions, the model's prediction seemed to be dictated by the presence of these words, ignoring the subtle context.

Our attempt to help the model focus more on the context and differentiate these subtle cases was to randomly mask some of the offensive words during training. The intuition was to encourage the model to attend to the context instead of specific terms by partially removing the dependency on certain words. During model training, when an input tweet contained words in the offensive word list ${ }^{7}$, we randomly replaced them with a $[P A D]$ token. We used the unmasked, original sample at inference time. We expected this procedure to help the model become more robust against different contexts of offensive word usage. Unfortunately, the masking strategy did not result in quantitative gains. A possible explanation for the performance decline could be that the naïve masking excessively deleted offensive words that were actually used offensively. A more balanced approach would be required to address this limitation in future work.

\section{Related Work}

Computational methods to identify offensive language have been studied in many different aspects (Davidson et al., 2017; Schmidt and Wiegand, 2017). Researchers have focused on a variety of categories of offensive language such as sexism, racism, and hate speech in general. Sap et al. (2019) identified a racial bias in hate speech detection datasets and proposed better annotation methods to address the problem. Park and Fung (2017) pointed out the gender bias in abusive language detection models and suggested a de-biasing algorithm. Qian et al. (2018) introduced a more fine-grained hate speech identification task than binary classification, and presented an architecture that leverages Conditional Variational Autoencoder (CVAE).

Large-scale pre-trained models like BERT have achieved state-of-the-art results in various NLP tasks

\footnotetext{
${ }^{7}$ https://www.cs.cmu.edu/ biglou/resources/, https://www.freewebheaders.com/full-list-of-bad-words-banned-by-google, https://github.com/LDNOOBW/List-of-Dirty-Naughty-Obscene-and-Otherwise-Bad-Words
} 
(Devlin et al., 2019; Yang et al., 2019). Wu and Dredze (2019), K et al. (2020), and Pires et al. (2019) explored the multilinguality of mBERT and confirmed the model's effectiveness in cross-lingual transfer. In the task of offensive language detection, (Liu et al., 2019) has adopted BERT to solve the problem. In our work, we also use mBERT but improve the method's ability under a multilingual setting by leveraging cross-lingual transfer.

\section{Conclusion}

In this paper, we describe NLPDove's approach to SemEval-2020 Task 12: Multilingual Offensive Language Identification in Social Media. The system yielded significant results in the competition for multiple languages, confirming the strength of large-scale pre-trained language models and the importance of various preprocessing schemes and model ensembling. We examine two data augmentation strategies and propose a new metric, TED, to quantify cross-lingual transferability. In qualitative analysis, we inspect factors why data selection with TED increased performance. We also present the limitations of our system, mainly failing to detect the speaker's intention expressed subtly in the context, hoping to offer valuable insights and elicit future work in the field.

\section{References}

Çağrı Çöltekin. 2020. A Corpus of Turkish Offensive Language on Social Media. In Proceedings of the 12th International Conference on Language Resources and Evaluation. ELRA.

Xilun Chen, Yu Sun, Ben Athiwaratkun, Claire Cardie, and Kilian Weinberger. 2018. Adversarial deep averaging networks for cross-lingual sentiment classification. Transactions of the Association for Computational Linguistics, 6:557-570.

Alexis Conneau and Guillaume Lample. 2019. Cross-lingual language model pretraining. In Advances in Neural Information Processing Systems, pages 7059-7069.

Thomas Davidson, Dana Warmsley, Michael Macy, and Ingmar Weber. 2017. Automated Hate Speech Detection and the Problem of Offensive Language. In Proceedings of ICWSM.

Jacob Devlin, Ming-Wei Chang, Kenton Lee, and Kristina Toutanova. 2019. BERT: Pre-training of deep bidirectional transformers for language understanding. In Proceedings of the 2019 Conference of the North American Chapter of the Association for Computational Linguistics: Human Language Technologies, Volume 1 (Long and Short Papers), pages 4171-4186, Minneapolis, Minnesota, June. Association for Computational Linguistics.

Jesse Dodge, Gabriel Ilharco, Roy Schwartz, Ali Farhadi, Hannaneh Hajishirzi, and Noah A. Smith. 2020. Fine-tuning pretrained language models: Weight initializations, data orders, and early stopping. ArXiv, $\mathrm{abs} / 2002.06305$.

Bryan Dosono and Bryan Semaan. 2019. Moderation practices as emotional labor in sustaining online communities: The case of aapi identity work on reddit. In Proceedings of the 2019 CHI Conference on Human Factors in Computing Systems, pages 1-13.

Antigoni Maria Founta, Constantinos Djouvas, Despoina Chatzakou, Ilias Leontiadis, Jeremy Blackburn, Gianluca Stringhini, Athena Vakali, Michael Sirivianos, and Nicolas Kourtellis. 2018. Large scale crowdsourcing and characterization of twitter abusive behavior. In Twelfth International AAAI Conference on Web and Social Media.

Karthikeyan K, Zihan Wang, Stephen Mayhew, and Dan Roth. 2020. Cross-lingual ability of multilingual bert: An empirical study. In International Conference on Learning Representations.

Olga Kovaleva, Alexey Romanov, Anna Rogers, and Anna Rumshisky. 2019. Revealing the dark secrets of BERT. In Proceedings of the 2019 Conference on Empirical Methods in Natural Language Processing and the 9th International Joint Conference on Natural Language Processing (EMNLP-IJCNLP), pages 4365-4374, Hong Kong, China, November. Association for Computational Linguistics.

Ping Liu, Wen Li, and Liang Zou. 2019. NULI at SemEval-2019 task 6: Transfer learning for offensive language detection using bidirectional transformers. In Proceedings of the 13th International Workshop on Semantic Evaluation, pages 87-91, Minneapolis, Minnesota, USA, June. Association for Computational Linguistics. 
Laurens van der Maaten and Geoffrey Hinton. 2008. Visualizing data using t-sne. Journal of machine learning research, 9(Nov):2579-2605.

Hamdy Mubarak, Ammar Rashed, Kareem Darwish, Younes Samih, and Ahmed Abdelali. 2020. Arabic offensive language on twitter: Analysis and experiments. arXiv preprint arXiv:2004.02192.

Ji Ho Park and Pascale Fung. 2017. One-step and two-step classification for abusive language detection on twitter. In Proceedings of the First Workshop on Abusive Language Online, pages 41-45, Vancouver, BC, Canada, August. Association for Computational Linguistics.

Telmo Pires, Eva Schlinger, and Dan Garrette. 2019. How multilingual is multilingual BERT? In Proceedings of the 57th Annual Meeting of the Association for Computational Linguistics, pages 4996-5001, Florence, Italy, July. Association for Computational Linguistics.

Zeses Pitenis, Marcos Zampieri, and Tharindu Ranasinghe. 2020. Offensive Language Identification in Greek. In Proceedings of the 12th Language Resources and Evaluation Conference. ELRA.

Georgios K. Pitsilis, Heri Ramampiaro, and Helge Langseth. 2018. Detecting offensive language in tweets using deep learning. CoRR, abs/1801.04433.

Jing Qian, Mai ElSherief, Elizabeth Belding, and William Yang Wang. 2018. Hierarchical CVAE for fine-grained hate speech classification. In Proceedings of the 2018 Conference on Empirical Methods in Natural Language Processing, pages 3550-3559, Brussels, Belgium, October-November. Association for Computational Linguistics.

Sarah T Roberts. 2014. Behind the screen: The hidden digital labor of commercial content moderation. Ph.D. thesis, University of Illinois at Urbana-Champaign.

Sara Rosenthal, Pepa Atanasova, Georgi Karadzhov, Marcos Zampieri, and Preslav Nakov. 2020. A Large-Scale Semi-Supervised Dataset for Offensive Language Identification. In arxiv.

Maarten Sap, Dallas Card, Saadia Gabriel, Yejin Choi, and Noah A. Smith. 2019. The risk of racial bias in hate speech detection. In Proceedings of the 57th Annual Meeting of the Association for Computational Linguistics, pages 1668-1678, Florence, Italy, July. Association for Computational Linguistics.

Anna Schmidt and Michael Wiegand. 2017. A Survey on Hate Speech Detection Using Natural Language Processing. In Proceedings of the Fifth International Workshop on Natural Language Processing for Social Media. Association for Computational Linguistics, pages 1-10, Valencia, Spain.

Rico Sennrich, Barry Haddow, and Alexandra Birch. 2016. Improving neural machine translation models with monolingual data. In Proceedings of the 54th Annual Meeting of the Association for Computational Linguistics (Volume 1: Long Papers), pages 86-96, Berlin, Germany, August. Association for Computational Linguistics.

Gudbjartur Ingi Sigurbergsson and Leon Derczynski. 2020. Offensive Language and Hate Speech Detection for Danish. In Proceedings of the 12th Language Resources and Evaluation Conference. ELRA.

Thomas Wolf, Lysandre Debut, Victor Sanh, Julien Chaumond, Clement Delangue, Anthony Moi, Pierric Cistac, Tim Rault, R'emi Louf, Morgan Funtowicz, and Jamie Brew. 2019. Huggingface's transformers: State-of-theart natural language processing. ArXiv, abs/1910.03771.

Shijie Wu and Mark Dredze. 2019. Beto, bentz, becas: The surprising cross-lingual effectiveness of BERT. In Proceedings of the 2019 Conference on Empirical Methods in Natural Language Processing and the 9th International Joint Conference on Natural Language Processing (EMNLP-IJCNLP), pages 833-844, Hong Kong, China, November. Association for Computational Linguistics.

Ellery Wulczyn, Nithum Thain, and Lucas Dixon. 2017. Ex machina: Personal attacks seen at scale. In Proceedings of the 26th International Conference on World Wide Web, pages 1391-1399.

Ruochen Xu and Yiming Yang. 2017. Cross-lingual distillation for text classification. In Proceedings of the 55th Annual Meeting of the Association for Computational Linguistics (Volume 1: Long Papers), pages 1415-1425, Vancouver, Canada, July. Association for Computational Linguistics.

Zhilin Yang, Zihang Dai, Yiming Yang, Jaime Carbonell, Russ R Salakhutdinov, and Quoc V Le. 2019. Xlnet: Generalized autoregressive pretraining for language understanding. In Advances in neural information processing systems, pages 5754-5764. 
Marcos Zampieri, Shervin Malmasi, Preslav Nakov, Sara Rosenthal, Noura Farra, and Ritesh Kumar. 2019 a. Predicting the Type and Target of Offensive Posts in Social Media. In Proceedings of the 2019 Conference of the North American Chapter of the Association for Computational Linguistics (NAACL), pages 1415-1420.

Marcos Zampieri, Shervin Malmasi, Preslav Nakov, Sara Rosenthal, Noura Farra, and Ritesh Kumar. 2019 b. Semeval-2019 task 6: Identifying and categorizing offensive language in social media (offenseval). In Proceedings of the 13th International Workshop on Semantic Evaluation, pages 75-86.

Marcos Zampieri, Preslav Nakov, Sara Rosenthal, Pepa Atanasova, Georgi Karadzhov, Hamdy Mubarak, Leon Derczynski, Zeses Pitenis, and Çağrı Çöltekin. 2020. SemEval-2020 Task 12: Multilingual Offensive Language Identification in Social Media (OffensEval 2020). In Proceedings of SemEval. 